\title{
A translocation breakpoint disrupts the ASPM gene in a patient with primary microcephaly
}

\author{
Bruno Pichon ${ }^{1,2,3}$, Sophie Vankerckhove ${ }^{3}$, Georges Bourrouillou ${ }^{4}$, Laurence Duprez ${ }^{1,2,3}$ \\ and Marc J Abramowicz*,1,3
}

\begin{abstract}
${ }^{1}$ Service de Génétique Médicale, Hôpital Erasme, Université Libre de Bruxelles, 808 Route de Lennik, 1070 Bruxelles, Belgium; ${ }^{2}$ Laboratoire de Cytogénétique, Hôpital Erasme, Université Libre de Bruxelles, 808 Route de Lennik, 1070 Bruxelles, Belgium; ${ }^{3}$ Laboratoire de Génétique Médicale et IRIBHM, Université Libre de Bruxelles, Belgium;

${ }^{4}$ CHU Purpan, F-31059 Toulouse, France
\end{abstract}

Primary microcephaly (microcephalia vera) is a developmental abnormality resulting in a small brain, with mental retardation. It is usually transmitted as an autosomal recessive trait, and six loci have been reported to date. We analyzed a translocation breakpoint previously reported in a patient with apparently sporadic primary microcephaly, at 1q31, where locus MCPH5 maps. The patient was lost to follow-up, and we sampled a maternal aunt who carried the familial translocation. FISH analyses showed that the insert of BAC clone RP11-32D17 spanned the breakpoint. The breakpoint was further located within a fragment of this insert corresponding to intron 17 of the ASPM gene, resulting in a predicted transcript truncated of more than half of its coding sequence. It is very likely that the proband carried a second ASPM mutation in trans, but he was not available for sampling and hence we could not confirm this hypothesis. Our observation adds to the mutation spectrum of ASPM in primary microcephaly, and is to our knowledge the second example of a constitutional, reciprocal translocation responsible for a bona fide autosomal recessive phenotype.

European Journal of Human Genetics (2004) 12, 419-421. doi:10.1038/sj.ejhg.5201169

Published online 3 March 2004

Keywords: brain development; Mendelian cytogenetics; MCPH5

\section{Introduction}

Microcephaly is a clinical finding usually defined as a head circumference smaller than 3 standard deviations below the mean for age and sex. It is referred to as primary microcephaly if nonsyndromic and after careful exclusion of craniosynostosis and of known causes of secondary microcephaly, for example, perinatal hypoxia. Primary microcephaly essentially reflects a small size of the underlying brain, comparable to that of early hominids, and

*Correspondance: Dr Marc Abramowicz, Service de Génétique Médicale, Hôpital Erasme, Université Libre de Bruxelles, 808 Route de Lennik, 1070 Bruxelles, Belgium. Tel: + 322 5556430; Fax: + 322 5556440;

E-mail: marcabra@ulb.ac.be

Received 31 October 2003; revised 16 December 2003; accepted 7 January 2004 results in mental retardation, typically not associated with other neurological deficits. ${ }^{1}$ Genetic defects causing primary microcephaly provide one approach to study this developmental disorder. These defects are usually transmitted as autosomal recessive traits (MCPH[251200]), with genetic heterogeneity, and six loci have been reported to date. $^{2,3}$ Among these, the MCPH5 locus has been reported in more than one ethnic population, ${ }^{2,4,5}$ and mutations of the ASPM gene have recently been found in MCPH5-linked microcephaly patients. ${ }^{6}$

Constitutional reciprocal translocations of autosomes are found in up to $0.1 \%$ livebirths. More than half are inherited and are not associated with an abnormal phenotype. ${ }^{7}$ Familial, reciprocal autosomal translocation segregating with a specific phenotype, although rare, has proved useful for characterizing genes involved in 
autosomal dominant disease. ${ }^{8}$ A familial and apparently silent reciprocal translocation is occasionally observed in a phenotypically abnormal propositus. From a theoretical viewpoint, such cases may result from mere chance, or reflect autosomal recessive inheritance, where one allele is disrupted by the translocation and the other allele by an intragenic mutation. ${ }^{9}$

Here, we report the molecular cytogenetic characterization of a balanced familial translocation segregating in a large family and previously reported in an infant with primary microcephaly. ${ }^{10}$ His parents were not consanguineous. The $\mathrm{t}(1 ; 4)$ translocation was inherited from his asymptomatic mother and was carried by at least five other unaffected family members over three generations. The 1q31-1q32.1 cytogenetic breakpoint was compatible with the MCPH5 locus linkage region, thus possibly disrupting one allele of the autosomal recessive MCPH5-linked microcephaly gene.

\section{Materials and methods}

The propositus was lost to follow-up and could not be reached in spite of extensive efforts. A lymphoblastoid cell line was then derived with informed consent from lymphocytes of a maternal aunt, carrying the translocation.

Metaphase spreads were G-banded using routine procedures. BAC clones spanning bands q31 and q32.1 of chromosome 1 were obtained from the Sanger Institute (http://www.sanger.ac.uk). Six BamHI digestion fragments of the RP11-32D17 insert (AL353809) were subcloned in pBluescript SK + vector (Stratagene, La Jolla, CA, USA). Identities of the subclones were checked by restriction analysis and direct sequencing.

For FISH analyses, all clones and subclones were labeled by incorporation of fluorescein-12-dUTP (Roche, Mannheim, Germany) using a standard nick-translation method. BAC probes were cohybridized on lymphoblastoid metaphase spreads with Spectrum Orange-labeled centromeric probes for chromosome 1, CEP 1 satII/III (Vysis, Downers Grove, IL, USA), and chromosome 4, CEP 4 (Vysis, Downers Grove, IL, USA). After hybridization, chromosome spreads were DAPI-counterstained and mounted in an antifading vectashield solution (Vector, Burlingame, CA, USA). Microscopy slides were analyzed with a Zeiss Axioskop epifluorescence microscope. Images were merged using a camera and CytoVision software (Applied Imaging, Newcastle upon Tyne, UK).

\section{Results and discussion}

The apparently balanced familial $\mathrm{t}(1 ; 4)$ (q31;p15.3) translocation, as present in lymphoblastoid cells, is shown in the partial karyotype, Figure 1a. BAC clones spanning the MCPH5 linkage region at $0.5-1 \mathrm{Mb}$ intervals were used as FISH probes on lymphoblastoid metaphase spreads. As shown in Figure 1c, one of these BAC probes, RP11-32D17, produced a FISH signal on both derivatives at 1q31 and 4 p15.3, whereas all the BAC probes with more telomeric inserts were translocated onto $4 \mathrm{p}$ (data not shown),

a

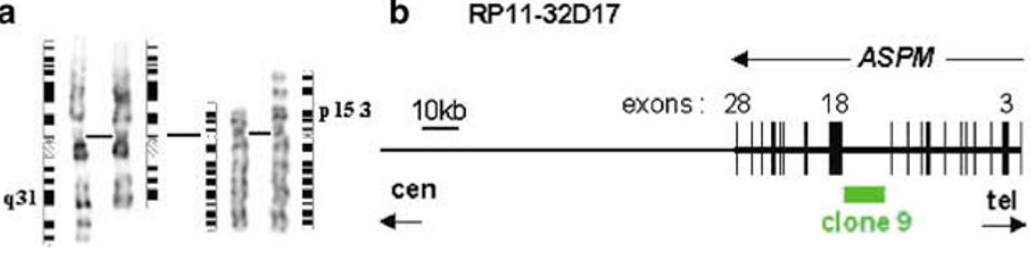

1 der1 4 der4

C

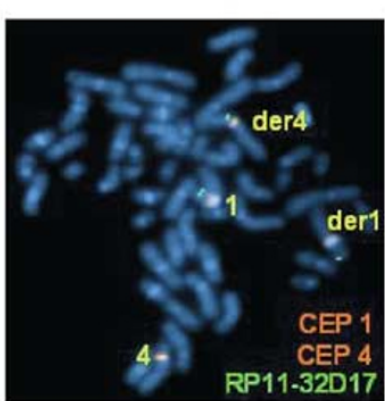

d

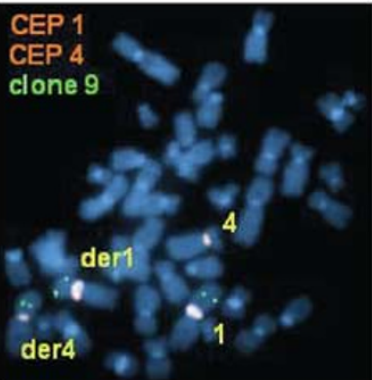

Figure 1 Molecular cytogenetics of the MCPH5 translocation breakpoint. (a) Partial karyotype and ideograms of $t(1 ; 4)(q 31 ; p 15.3)$. (b) Insert of the RP11-32D17 BAC clone showing the 28 exons of the ASPM gene and clone 9, a subcloned BamHI restriction fragment, used as FISH probe in (d). (c) RP11-32D17 (green) hybridization onto chromosome 1, derivative 1 (der1) and derivative 4 (der4). (d) Clone 9 (green) hybridization onto chromosome 1, der1 and der4. CEP1 (red) and CEP4 (red) (Vysis) probes hybridize to centromere 1 and centromere 4, respectively. 
indicating that RP11-32D17 encompasses the translocation breakpoint. The $168 \mathrm{~kb}$ genomic insert of the latter BAC contains the ASPM gene in its telomeric portion (Figure 1b). In order to locate the breakpoint more precisely, six BamHI digestion fragments of the RP11-32D17 BAC clone were used as FISH probes. One of these probes, clone 9, containing part of intron 17 of the ASPM gene (Figure 1b), produced a FISH signal on both derivatives, as shown in Figure 1d. All the subcloned probes with more centromeric inserts hybridized onto derivative 1 only (data not shown). These data locate the translocation breakpoint within intron 17 of the ASPM gene, which results in a predicted protein truncated of more than half of the primary sequence, including most of the IQ domains. Such a truncating mutation is predicted to produce a null allele, and is consistent with the truncating point mutations reported in primary microcephaly patients. ${ }^{6,11}$

The balanced $t(1 ; 4)$ translocation characterized here segregates in at least five asymptomatic subjects over three generations, ${ }^{10}$ consistent with recessivity and uncovering of a mutation of the second allele in the microcephalic propositus. Although he and his father, who presumably carried a point mutation of the second allele, were lost to follow-up and could not be sampled for DNA analysis, we believe our data indicate bona fide autosomal recessive transmission of primary microcephaly. To our knowledge, this is the second example of an autosomal recessive disease associated with gene disruption by a reciprocal translocation. ${ }^{9}$

\section{Acknowledgements}

CG Woods informed us of ASPM identification ahead of his publication (Bond et al., 2002). This research is supported by the
Belgian FRSM, the Fonds de recherche clinique Erasme, and the Fonds Forton. We thank S Delbauve and B Marredda for expert technical assistance.

\section{References}

1 Mochida GH, Walsh CA: Molecular genetics of human microcephaly. Curr Opin Neurol 2001; 14: 151-156.

2 Roberts E, Hampshire DJ, Pattison L et al: Autosomal recessive primary microcephaly: an analysis of locus heterogeneity and phenotypic variation. J Med Genet 2002; 39: $718-721$.

3 Leal G, Roberts E, O Silva EO, Costa SMR, Hampshire DJ, Woods CG: A novel locus for autosomal recessive primary microcephaly (MCPH6) maps to 13q12.2. J Med Genet 2003; 40: 540-542.

4 Jamieson CR, Fryns JP, Jacobs J, Matthijs G, Abramowicz MJ: Primary autosomal recessive microcephaly: MCPH5 maps to 1q25-q32. Am J Hum Genet 2000; 67: 1575-1577.

5 Pattison L, Crow YJ, Deeble VJ et al: A fifth locus for primary autosomal recessive microcephaly maps to chromosome $1 \mathrm{q} 31$. Am J Hum Genet 2000; 67: 1578-1580.

6 Bond J, Roberts E, Mochida GH et al: ASPM is a major determinant of cerebral cortical size. Nat Genet 2002; 32: 316-320.

7 Jacobs PA: Structural rearrangements of the chromosomes in man. in Hook EB, Porter IH (eds) Population Cytogenetics. New York: Academic press; 1977, pp 81-87.

8 Bugge M, Bruun-Petersen $\mathrm{G}$, Brondum-Nielsen $\mathrm{K}$ et al: Disease associated balanced chromosome rearrangements: a resource for large scale genotype-phenotype delineation in man. J Med Genet 2000; 37: 858-865.

9 Hearn T, Renforth GL, Spalluto C et al: Mutation of ALMS1 a large gene with a tandem repeat encoding 47 amino acids, causes Alstrom syndrome. Nat Genet 2002; 31: 79-83.

10 Perez-Castillo A, Martin-Lucas MA, Abrisqueta JA: Is a gene for microcephaly located on chromosome 1? Hum Genet 1984; 67: 230-232.

11 Bond J, Scott S, Hampshire DJ et al: Protein-truncating mutations in ASPM cause variable reduction in brain size. Am J Hum Genet 2003; 73: 1170-1177. 